\title{
Interview Herzkrank auf Reisen
}

Weder eine Herzklappenerkrankung noch ein kürzlich durchgemachter Myokardinfarkt schließen prinzipiell eine Reise aus. Es kommt darauf an, wie stabil und belastbar der Patient ist, erklärte Prof. Dr. Markus Flesch vom Marienkrankenhaus in Soest im Interview.

Chronisch herzkranke Patienten, die auf Reisen gehen, sollen sich zuvor beim Hausarzt untersuchen lassen. Welche Fragen gilt es da zunächst zu stellen? Flesch: Es reichen einfache Fragen um zu klären, wie belastbar ein Patient ist. Beispielsweise: Können Sie ein Stockwerk Treppen steigen? Oder: Welche Wegstrecke können Sie im Flachen zurücklegen? Ansonsten gehören dazu die üblichen Fragen wie bei jeder Anamnese, also nach Luftnot, Angina pectoris, Palpitationen oder Herzrasen. Daraus ergeben sich Hinweise darauf, ob der Herzpatient in einem stabilen oder in einem instabilen Zustand ist. Nicht reisefähig ist ein $\mathrm{Pa}-$ tient im Stadium der kardialen Dekompensation oder ein Patient, unmittelbar nachdem er ein akutes Koronarsyndrom durchgestanden hat.

Was gilt allgemein für die Flugfähigkeit? Flesch: Flugfähig ist, wer ein Stockwerk Treppensteigen ohne Mühe meistert. Ein objektiveres Kriterium ist eine fahrradergometrische Belastbarkeit bis 50 Watt. Es ist aber keineswegs erforderlich und

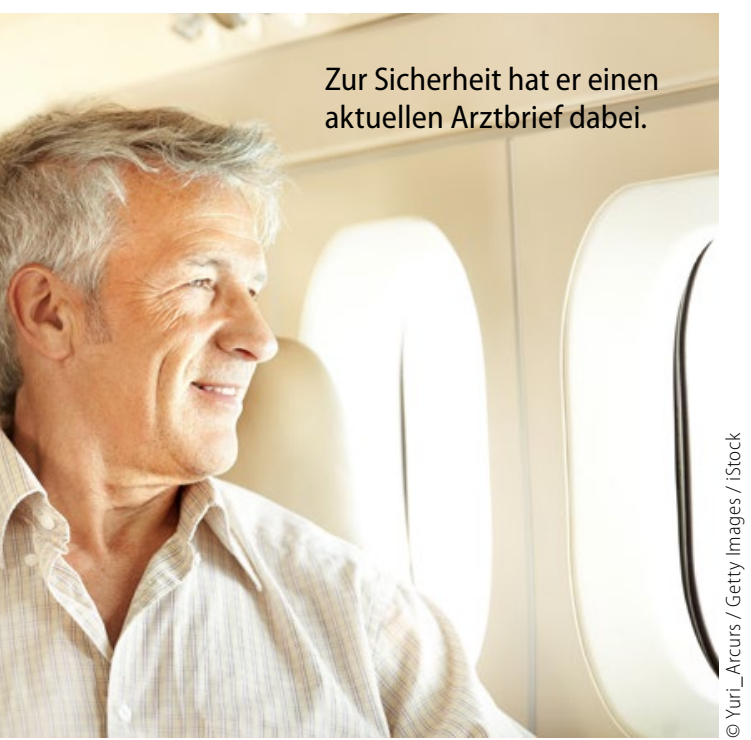

logistisch umsetzbar, bei jedem (flug) reisefreudigen Patienten vor der Reise ein Belastungs-EKG zu machen.

Was sollte man bei Patienten mit Herzklappenerkrankungen beachten?

Flesch: Weder eine Aortenklappenstenose noch eine Aortenklappeninsuffizienz oder entsprechende Mitralklappenerkrankungen sind prinzipiell Hindernisse für eine Reise. Hier gilt das Gleiche wie für die Herzinsuffizienz oder koronare Herzkrankheit: Wer eine belastende Reise antritt, sollte im Stadium der Kompensation sein.

Extrem vorsichtig sein muss man allerdings, wenn eine Flugreise angetreten werden soll oder Reisen in hoch gelegene Regionen geplant sind. Hier ist insbesondere darauf zu achten, dass beim Patienten keine ausgeprägte pulmonale Hypertonie besteht.

Sollten Patienten, die Vitamin-K-Antagonisten einnehmen, in der Lage sein, selbstständig ihren INR zu messen und die Dosis anzupassen?

Flesch: Das ist in der Tat eine Limitation für Patienten, die auf Vitamin-K-Antagonisten eingestellt sind. Denn im Allgemeinen ist es mit üblichen touristischen Aktivitäten wenig vereinbar, Gerinnungskontrollen durchführen und die Dosis anpassen zu lassen. Bei stabilem INR ist das Risiko gering. Bei instabilem INR oder Quick-Wert ist natürlich prinzipiell und unabhängig von einer Reise zu überlegen, ob nicht eine alternative Antikoagulation mit neueren oralen Antikoagulanzien infrage kommt. Wichtig ist der Hinweis für die Patienten, dass die tägliche Vitamin-K-Zufuhr mit der Nahrung, also über Salate und grünes Gemüse, ungefähr gleich zu halten ist, wie es zu Hause der Fall ist. Wer davon im Urlaub vergleichsweise mehr isst, braucht auch eine höhere Marcumar-Dosis.
Die gewohnten Einnahmezeiten einzuhalten, ist bei Flügen über mehrere Zeitzonen nicht möglich...

Flesch: Wegen der sehr langen Halbwertszeit des hierzulande üblichen Phenprocoumons ist das eigentlich kein Problem.

Wie sieht es mit Empfehlungen zur Thromboembolieprophylaxe aus?

Flesch: Die wichtigste Empfehlung an Reisende, egal ob im Bus, im Flugzeug, in der Bahn oder im eigenen Auto, ist erstens, ausreichend zu trinken und zweitens, sich immer wieder etwas $\mathrm{zu}$ bewegen. Selbst im Flugzeug sollte man also immer wieder aufstehen, im Gang auf und ab gehen. Wer kann, sollte auch mal eine Kniebeuge machen. Wichtig ist es, die Muskelpumpe in den Waden regelmäßig zu betätigen, um eine Thrombose zu vermeiden.

Im Übrigen schützt auch die Business Class nicht vor einer Thrombose. Günstig ist in diesem Zusammenhang, dass viele thrombosegefährdete Patienten oder Patienten nach bereits stattgehabter Thrombose und Lungenembolie heute dauerhaft antikoaguliert sind. Ist das nicht der Fall und steht eine Reise mit längerer Immobilisation an, würde ich zu einer Thromboseprophylaxe mit einem oralen Antikoagulans oder mit niedermolekularem Heparin raten. Studien, die uns hier empirische Sicherheit bieten, gibt es leider nicht. Der den Reisenden beratende Arzt muss pragmatisch vorgehen.

Welche Unterlagen zur Krankheit empfehlen Sie mitzugeben?

Flesch: Es ist sinnvoll, wenn chronisch kranke Patienten einen aktuellen Arztbrief mit Diagnosen und Medikation bei sich haben. Eine Medikamentenliste wird mittlerweile allen Patienten von der Hausarztpraxis ausgehändigt.

Viele Ärzte sind zudem bereit, dem Patienten einen Arztbrief in englischer Sprache mit auf die Reise zu geben. Bisweilen ist das auch bei der Einreise in ein Land mit Zollkontrolle hilfreich. 